\title{
Reducing Routing Overhead in Random Walk Protocol under MP2P Network
}

\author{
Ajay Arunachalam, Ohm Sornil \\ Department of Computer Science, School of Applied Statistics, National Institute of Development Administration, \\ Bangkok, Thailand
}

\begin{tabular}{l}
\hline \hline Article Info \\
\hline Article history: \\
Received Sep 7, 2015 \\
Revised Nov 9, 2015 \\
Accepted Nov 23, 2015 \\
\hline
\end{tabular}

Keyword:

MANET

Mobile peer-to-peer

MP2P

Peer-to-peer

Random walk

Resource discovery

Search

\begin{abstract}
Due to network dynamics in self-organizing networks the resource discovery effort increases. To discover objects in unstructured peer-to-peer network, peers rely on traditional methods like flooding, random walk and probabilistic forwarding methods. With inadequate knowledge of paths, the peers have to flood the query message which creates incredible network traffic and overhead. Many of the previous works based on random walk were done in wired network. In this context random walk was better than flooding. But under MANETs random walk approach behaved differently increasing the overhead, due to frequent link failures incurred by mobility. Decentralized applications based on peer-to-peer computing are best candidates to run over such dynamic network. Issues of P2P service discovery in wired networks have been well addressed in several earlier works. This article evaluates the performance of random walk based resource discovery protocol over P2P Mobile Adhoc Network (MP2P) and suggests an improved scheme to suit MANET. Our version reduces the network overhead, lowers the battery power consumption, minimizes the query delay while providing equally good success rate. The protocol is validated through extensive NS-2 simulations. It is clear from the results that our proposed scheme is an alternative to the existing ones for such highly dynamic mobile network scenario.
\end{abstract}

Copyright $\odot 2016$ Institute of Advanced Engineering and Science. All rights reserved.

\section{Corresponding Author:}

Ajay Arunachalam,

Department of Computer Science, School of Applied Statistics,

National Institute of Development Administration,

Bangkok, Thailand.

Email: ajay.abhilash.28@gmail.com

\section{INTRODUCTION}

MANETs are multihops dynamic network which are characterized by wireless links, mobile nodes and multihop routing. In such network the nodes that are not directly connected at Layer 2 can communicate through Layer 3 routing. Due to continous movement of nodes, resource discovery in such networks become more challenging. Flooding and Random walk [1] are two typical search technique. Flooding is simple and widely accepted method wherein the query is flooded to every node in the network. While in Random walk the query is forwarded randomly to some nodes in the network. Even though flooding and Random walk have their own limitations these methods have to be eventually considered to suit such self-organizing multihop networks.

Performance of P2P resource discovery in wired networks have been well researched in the past. Different content searching in such P2P network were evaluated over wired context and fixed layout like the internet. But their results are not feasible to be validated against MANETs, due to deficiency of infrastructure, mobility, energy consumption issues and churn. [2] summarizes the problems related to service discovery protocols in MANETs. They study random walk protocol over such multi-hop network. 
Further they list out several issues pertaining to random walk with major of those being next-hop selection criteria, valid termination check parameter, multiple query walk, unicast transmission disadvantage etc. A brief survey of various searching techniques for wireless adhoc networks is given in [3]. Peer-to-Peer networks are popular and such systems constitute majority of internet traffic [4] due to its flexible and distributed network characteristics. Over the past decades P2P architecture based systems have been deployed over internet to provide services mainly including file sharing, telephony, information retrieval system, torrent and video conferencing applications etc. P2P networks are broadly classified based on its design as structured and unstructured architecture. In structured P2P networks the peers strictly are wellorganized with each peer maintaining an DHT index.

The famous examples being CAN, Chord, Pastry,Tapestry. Since in these system participating peers position is structured so the query search is more efficient. But these system have limitations in mobile environment increasing the complexity and overhead as continuous modification of index pointer is needed to be done because of rapid mobility. On the contrary, in unstructured networks there is no strict regulation for formation of topology and peer positioning. Further there is no need of any central indexing for placement of the contents. Nodes can join and leave randomly. Among unstructured P2P protocols the most referred ones are Gnutella, Fastrack, Kazaa, Freenet etc. When we say P2P it means each peer has equal role in the network for a fully distributed P2P system. Even today major mobile communication architectures are mainly centralized. One of the best possibilities to overcome the bottleneck caused due to centralized architecture is to setup a P2P platform [5]. This communication pattern can suit any network including wired, wireless and even MANETs. Incorporating peer-to-peer network characteristics in mobile adhoc networks is $\mathrm{P} 2 \mathrm{P}$ MANETs or Mobile P2P (MP2P) network. Due to similarities in P2P and MANETs, a P2P Overlay can run over Mobile Adhoc Network. But at the same time their combination also poses difficulties due to difference in the operating layer, transmission mechanism and rapid mobility in MANETs [6-7].

We briefly discussed about MANETs and P2P networks and general difficulties in adopting P2P over MANET. The rest of the paper is organized as follows. In Section 2, we review the related work for how resource discovery is carried in Peer-to-Peer and Mobile Peer-to-Peer networks. Section 3 presents our proposed resource discovery protocol. Section 4 talk about the simulation environment and shows the experimental results and finally Section 5 concludes with a summary.

\section{BACKGROUND AND RELATED WORK}

This Section discusses about different version of the Random walk protocol in wired and wireless network scenario. It also gives a brief about various resource searching methods in past.

Resource discovery in pure P2P system is to discover the IP address of the node that has the resource. Traditionally blind and knowledge based search techniques are used in unstructured P2P network. In such network peers churn randomly. Two traditional resource searching techniques are preferred for such environment i.e. flooding and random walk where the query request are forwarded blindly to the neigbhors without any prior knowledge. Many variations of flooding method were also proposed such as modified BFS [8], iterative deepening [9] etc.

In [10] a controlled flooding based search strategy for unstructured P2P network is proposed. Here the probability of forwarding the query to the selected neigbhor is based on the number of connected nodes and the distance metric calculated from the query to initiator node. But here we feel that the distance metric calculation itelf is an overhead with a need of controller node and further the nodes should have GPS like functionality enabled to get the positions in wireless network. Various improved version of random walk were also proposed in the past. This technique can't learn from their past experiences due to the random forwarding nature of the query. Search mechanism based on such probabilistic nature also dwell in the past that were proposed to improve the overall performance which include k-walker random walk [11], two-level random walk [12], biased random walk [13] etc. Such random walk based search mechanism is highly beneficial in wired context [14].

Alternatively resource discovery protocols with a mixture of both deterministic and probabilistic nature were also proposed to increase the search efficiency. Several methods were also proposed which included the characteristics of local flooding with independent k-walkers. Starting with flooding the search is extended to k-walker's until the query is resolved. In [15-16] an alternative way with a combination of simple and tree-based flooding was proposed which had high tradeoff with respect to the cost incurred for maintaining the overall tree like sub structure. To overcome this drawback the authors of [17] proposed an dynamic search protocol that takes the advantage of flooding and random walk approach based on context of the search, along with an mixture of feedback from the previous search results [18] proposed an bio-inspired swarm intelligence based resource discovery protocol for unstructured P2P network. Their work is based on the ideology of nature inspired elephant's journey for food from one place to another. An improvement of the 
APS search method was proposed in [19]. They use the ant colony optimization technique considering both the file types and score obtained utilizing the past search history associated for each file type.

In $\mathrm{P} 2 \mathrm{P}$ network the concepts of semantic ontology and heirarchical clustering have also been used in the past to improve the search efficiency [20]. To lower the redundancy and construct an effective knowledge based routing table in Gnutella-flooding protocol they introuduce the theory of semantic ontology to build a meaningful relationship between the query request and the reply. In such networks the nodes churn frequently and further pose potential security threats by intrusion of malicious nodes. The concept of node reputation [21] are used to alleviate threats caused by such nodes that overall reduces the network performance. But for a Mobile P2P network due to mobility further it rules out the possibility of applying informed search method as knowledge derived from the past experience will not be always feasible in such dynamic scenario. So improved resource discovery protocols based on characteristics of Mobile Peer-to-Peer networks have to be given attention as compared to the traditional P2P protocols. While blind searching approach is eventually considered, it increases the query message cost drastically. Self-organizing networks such as MANET and P2P poses additional challenges as compared to wired infrastructure. Most resource discovery protocols use flooding approach as basic style for searching. Suitability of random walk for MANETs is also studied in past. An improved search algorithm for unstructured peer to peer overlay network is proposed in [22]. They improve random walk with ' $m$ ' multiple walker's and include replication with 2-hop jump mechanism, but then their proposed search technique is based on super peer architecture. Every walker can jump to randomly selected 2-hop neighor super peer. Further in their work peers use cache mechanism to enhance the search efficiency.

A different resource discovery approach for unstructured Mobile P2P network is discussed in [23]. They proposed an search scheme that uses time stamp to validate the freshness of the resource information of the neigbhor peers. Their protocol also is based on super peer structure. The resource information transmitted from the neigbhor peers are prioritized using ranking function that considers the interest and moving direction of the peer [24] propose an self-aware search protocol where initially the ranking of nodes is done based on positive resource reply mechanism and then among such nodes those that have common subject of interest form one group. For every such group a super node is associated which is responsible for communication between other different groups. A simple resource discovery algorithm based on super node for Mobile P2P network is presented in [25]. The basic idea of their work is to choose the node with high capacity and week mobility as super peer and allow such peer to transmit query quickly.Here the flooded request model is used to forward query among every other super peer. An interesting way for resource discovery in Mobile Peer-to-Peer network is tried in [26].

Instead of forwarding the query message to every node or set of nodes the author uses the concept of Reverse Nearest Neigbhor (RNN). An RNN query returns all objects that considers the query object as their nearest neigbhor. They proposed an algorithm that makes use of all information from the peers, where the search is progressed within the boundary polygon being constructed by those peers that were involved in construction of the polygon. [27] focuses on membership management in unstructured Mobile P2P network. Due to churn, dynamic topology of MANETs and with scare environment the P2P membership management becomes very difficult. They suggest a scheme for joining and leaving the membership tree also while considering the node mobility. In [1] the performance of the random walk protocol is improved by replication of the query at multiple instances and adding clustering method at the Overlay network. An innovative method is proposed in [28] based on the random walk technique, where the transmission range is doubled by enhancing the power thereby increasing the range for query forwarding. The efficiency of P2P content discovery protocol on Mobile Adhoc Network have been evaluated in [29]. They conclude their study with an critical summary which suggests that unstructured protocols work better under MANET. We noticed that mostly all the resource search schemes proposed for Mobile Peer-to-Peer environment were mainly based around an super peer architecture, i.e, utilized super nodes to process the query [22]-[25], [30]-[36]. In some of the above discussed work the resource discovery structure itself is complex. We aim to present a light weight resource search design technique by using the wireless network characteristics. We focus on the fully decentralized pure P2P network architecture i.e. our proposed scheme is not bound around any special node. We propose a search algorithm for a flat topology that should work without any hierarchy information.

\section{PROPOSED PROTOCOL}

In this Section, we give a brief overview of our unstructured resource discovery protocol. Most of the existing unstructured P2P protocols used flooding and random walk mechanism to resolve a query. So to begin with we first evaluated these techniques over MANET and studied their performance strictly under such dynamic network scenario [37]. In our observation we suggested that any improvement to be done should consider the underlay topology to compliment the MANET network characteristics. And also further 
to improve the search efficiency we need to minimize the message duplication. For unstructured P2P network, resource discovery as in [38] visited more number of peers within less time and generate less query message. While on the contrary in our technique we enhance the search performance which is carried out by visiting less number of actual peers within minimum duration. In our approach to select a random neighbor for forwarding the query request, a probabilistic approach is used. The normal unicast based random walk technique under MANET has several issues in terms of routing overhead, battery power consumption, search delay, search efficiency etc. mainly due to the frequent topology change. To address this we propose a random walk method with a mixture of conditional addressing and message delivery technique.

\subsection{Search Mechanism}

For efficient and quick resource search we suggest an conditional addressed broadcast based random walk technique to minimize the search delay and utilize the network resources effectively. Generally neighbor nodes are detected by doing 1 hop broadcast hello protocol. To establish a connection with existing nodes the newly arrived node broadcast the HELLO message within 1-hop transmission range. The other nodes upon receiving HELLO message replies with an HELLO RESPONSE message. In this way the neighor list is updated frequently in the overlay. But doing it the traditional way will again increase the broadcast overhead. So we use the neighbor information which is available with AODV [39] routing protocol and get this information from the P2P application. Now to resolve a request, the query initiator node selects a random neighbor from the neighbor list that is got from the routing layer neighbor list information. The node first checks if the query is already seen. If seen before, it drops the query.

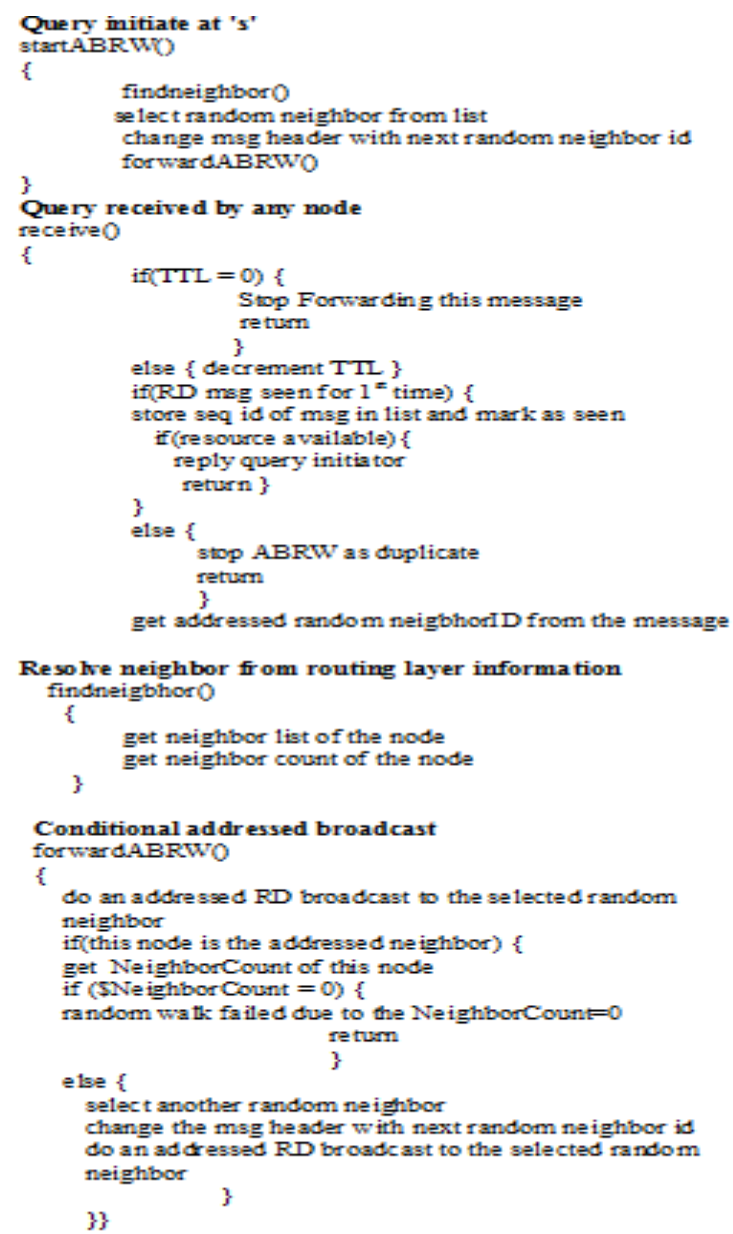

Figure 1. Addressed Broadcast Random Walk Search Algorithm (ABRW)

The message is then forwarded to the selected random neighbor over an addressed broadcast communication which means the message is 1-hop broadcast to a particular node wherein the addressed 
neighbor node will process it on normal condition, but all the other nodes within that range can see that message while further the random walk will only be resumed by that particular addressed node. In case the resource node is within the transmission range then it may listen that message and thereby even without waiting for getting the message through the normal random walk that is in process the query can be resolved intermediately. The proposed approach continues this search process until query is solved or TTL expires. Here the query is not flooded to all the nodes in the network. Further the probability of resolving the initiated query is high, because the query can be resolved by intermediate neighbors in case they have the requested resource. The network overhead will also get reduced since the message is just broadcasted to 1-hop. Our method is not at all using any routing mechanism while forwarding the message, so the mobility of the node and the link failures at routing layer will not affect the resource discovery process. In our method the duplicate requests that may be received over several another paths are eliminated that hinders the search efficiency. Our proposed scheme is given as an algorithm in Figure 1.

\section{PERFORMANCE EVALUATION}

In this Section, we evaluate the performance of the resource discovery protocols. We carried out the experiments in NS2 v 2.35 for 60, 70, 80 and 90 nodes with transmission range as $250 \mathrm{~m}$. The nodes moved according to the random way point model. The node speed is $20 \mathrm{~m} / \mathrm{s}$ and lookup interval is $5 \mathrm{sec}$. The rest of environment, network scenario and P2P application related parameters are the same as in [40].

\subsection{Evaluated metrics}

To verify the performance of resource discovery algorithms under consideration we evaluate it for the following metrics. (1) Success rate (2) Response time (3) P2P Network Overhead (4) Overall Dropped Packets (5) Avg Consumed Energy of P2P Network (6) P2P Agent Level Hop to Hop Delay (7) P2P Agent Level Throughput. We present the definition of the metrics as follows:

\subsubsection{Success Rate}

The success rate is an important metric for measuring the performance of P2P resource discovery algorithm. It is a ratio of total generated requests and the successfully received replies. It is measured in percentage as follows:

$$
\text { Success Rate }=(\operatorname{Rep} / \operatorname{Req}) * 100(\%)
$$

where

Req is the total generated resource requests and

Rep is the total successfully received resource replies

The higher success rate of resource discovery signifies the better performance of the resource discovery algorithm.

\subsubsection{Avg Response Time}

The response time is also another important metric for measuring the performance of $\mathrm{P} 2 \mathrm{P}$ resource discovery algorithm. It is the time difference between the resource request generation time and the resource reply received time. It is calculated as follows:

Response time $=\mathrm{T}_{\text {Rep }}-\mathrm{T}_{\text {Req }}(\mathrm{sec})$ algorithm

The lower resource discovery time signifies the better performance of the resource discovery

\subsubsection{P2P Network Overhead}

In this work we measure Overhead in terms of total number of generated and forwarded routing messages at the network layer.

\subsubsection{Overall Dropped Packets}

Packet drop in a MANET scenario is an important metric to evaluate the network performance. Generally packets will be dropped due to several reasons in a wireless adhoc network scenario. And particularly this dropping of packets in a typical P2P network will expected to be high since these algorithms will use lot of duplicates of the same resource discovery message during the entire resource discovery 
process that will increase the overhead and causing network bottle neck. We count the Packets Dropped at all the network layers of all the P2P nodes.

\subsubsection{Avg Consumed Energy of P2P Network}

Generally the Consumed Energy is calculated as the average energy consumed by all the nodes of the network for the entire time of the simulation. It is measured in Joules. It is calculated as follows:

$$
E_{\text {Avg }}=\frac{1}{N} \sum_{i=1}^{N} I E_{i}-F E_{i} \quad \text { (in Joules) }
$$

where

$\mathrm{E}_{\mathrm{Avg}}$ is the calculated Avg Consumed Energy of P2P Network in Joules

$\mathrm{N}$ is the total number of nodes in the P2P network

$\mathrm{IE}_{\mathrm{i}}$ is the initial battery Energy of the Node I

$\mathrm{FE}_{\mathrm{i}}$ is the final battery Energy of the Node I

\subsubsection{P2P Agent Level Hop to Hop Packet Delay}

This metric is a refined version of normal delay calculation. Here instead of calculating delay between source and destination, it is calculated for each packets generated during the each of the search process. It is the time taken for a packet to reach each hop during forwarding the resource discovery request. It is calculated as follows:

$$
\text { Hop to Hop Packet Delay }=\mathrm{T}_{\mathrm{r}}-\mathrm{T}_{\mathrm{f}} \quad \text { (in ms) }
$$

where

$\mathrm{T}_{\mathrm{r}}$ is the time at which a node receives a resource discovery request and

$\mathrm{T}_{\mathrm{f}}$ is the time at which that resource discovery request was forwarded from the previous hop.

\subsubsection{P2P Agent Level Throughput (kbps)}

Normalized throughput is a measure of number of messages or data packets successfully delivered over time. It is generally measured in Kbps or Mbps. So higher value will signify better performance. Here the P2P Agent Level Throughput is calculated as folows:

$$
\text { Throughput }=\mathrm{A}_{\mathrm{rs}} / \mathrm{T}_{\mathrm{d}} \quad(\text { in } \mathrm{kbps})
$$

where

$\mathrm{A}_{\mathrm{rs}}$ is the sum of all the packet sizes received at the P2P Agent Level of all the nodes in the network. $\mathrm{T}_{\mathrm{d}}$ is the total time duration of the $\mathrm{P} 2 \mathrm{P}$ resource discovery scenario

\subsection{Results and Discussion}

We choose two basic protocols i.e. flooding and random walk for comparison with our proposed technique. Figure 2 shows the successful queries measured as a ratio of total generated requests and successful replies for entire duration of the simulation. The performance of our proposed technique is equal to that of the flooding method. The random walk technique performs poorly.

In Figure 3, query response time is used as metric to validate the efficiency of the resource discovery protocol. The query response time is measured as the shortest time difference the protocol takes for the query to be resolved. The algorithm with least query delay signifies the best method. Our proposed method performs better than the existing methods. 


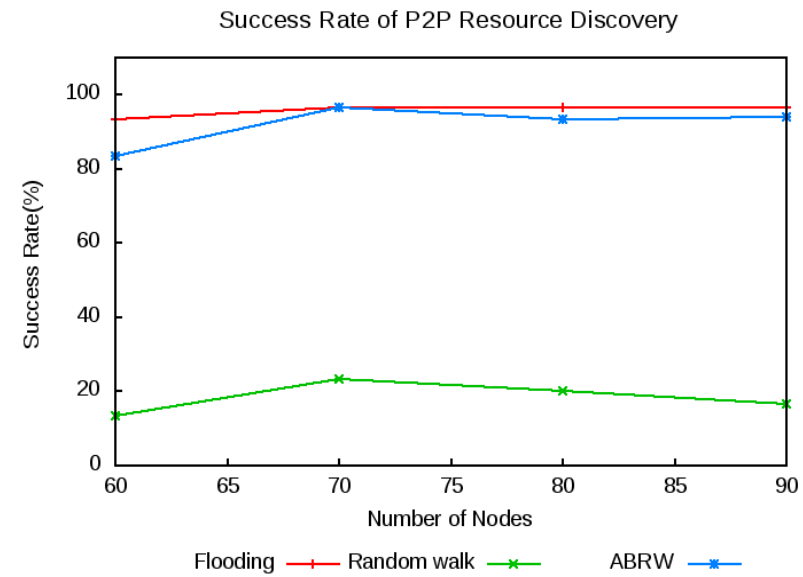

Figure 2. Query Success Rate

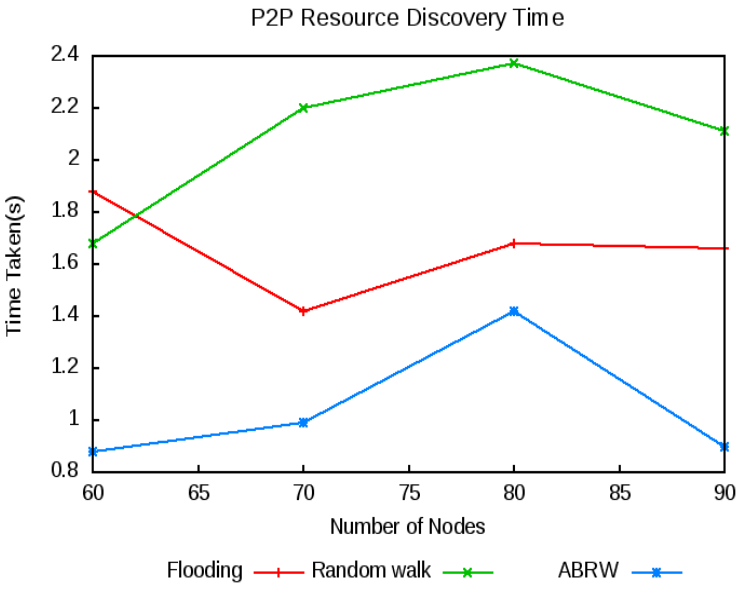

Figure 3. Search Response Time

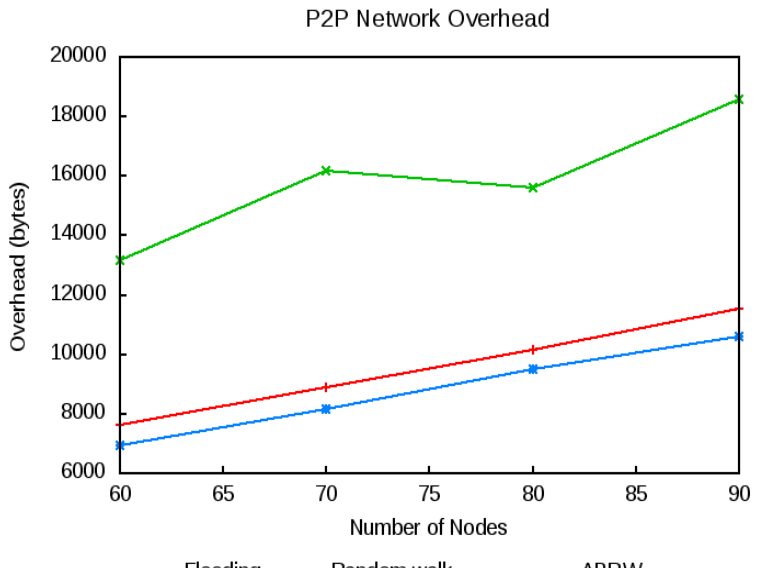

Flooding $\longrightarrow$ Random walk $\rightarrow \quad$ ABRW $\rightarrow-$

Figure 4. Overhead Generated by P2P Network

The Figure 4 shows the comparison of the Network Overhead for our proposed and the existing techniques. We measure the overhead in terms of generated routing packets. The overhead for our protocol is very low as compared to others. The reason for this is the use of conditional addressing. In random walk protocol the overhead is high due to unicast message transmission.

We display the results of dropped packet count in Figure 5. We measure the overall packet dropped metric as the difference between the number of packets send and number of packets received. In this case the total number of packets dropped during the simulation is measured. The graph shows that the performance of our proposed technique is better than all existing resource discovery protocols. Our proposed protocol performs better due to the message forwarding design which unaffects the resource discovery process.

Figure 6 displays the performance of various protocols in terms of energy consumed. The mobile nodes in MANETs are battery operated and hence power saving becomes very important. As seen in the graph our proposed protocol consumes less battery power than the existing methods. The random walk protocol performs poorly.

In Figure 7 hop-based packet delay is measured to evaluate the performance of the P2P protocol. Only the data packets that are successfully delivered are considered for each resource discovery process. The random walk performs poorly. Our proposed protocol shows good results. 


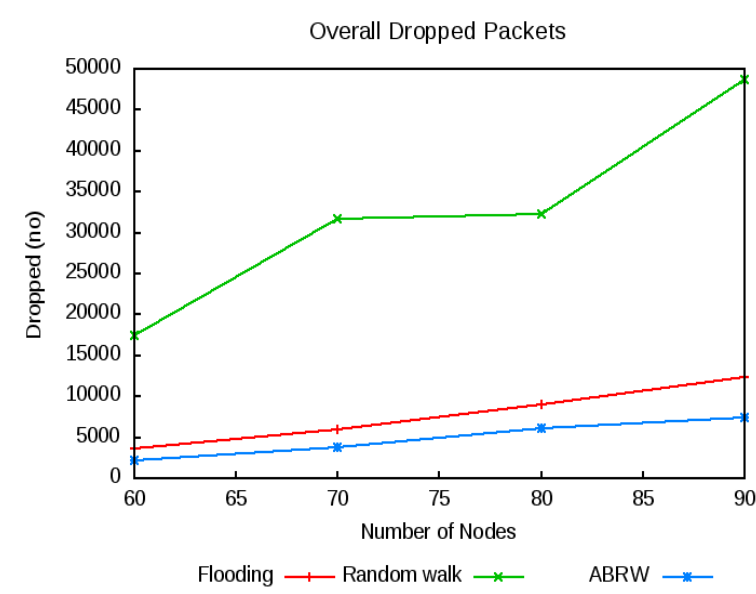

Figure 5. Dropped packets count

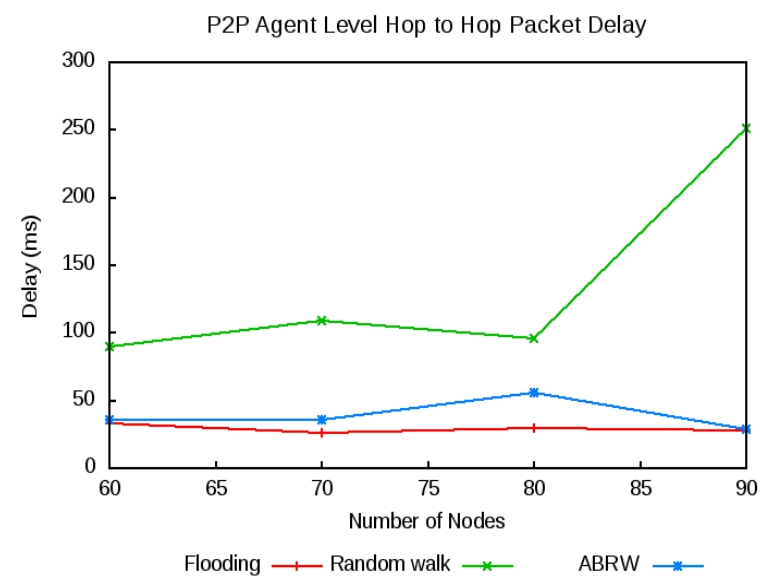

Figure 7. Hop to Hop Packet Delay

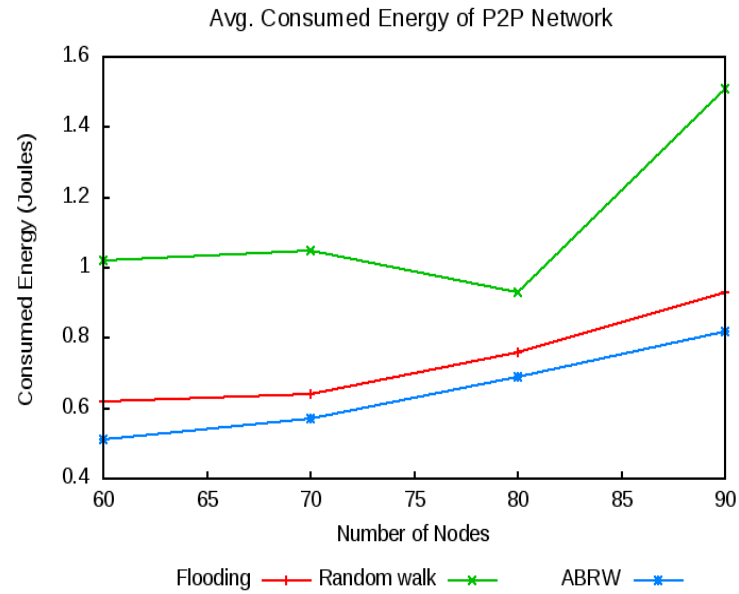

Figure 6. Battery power consumption

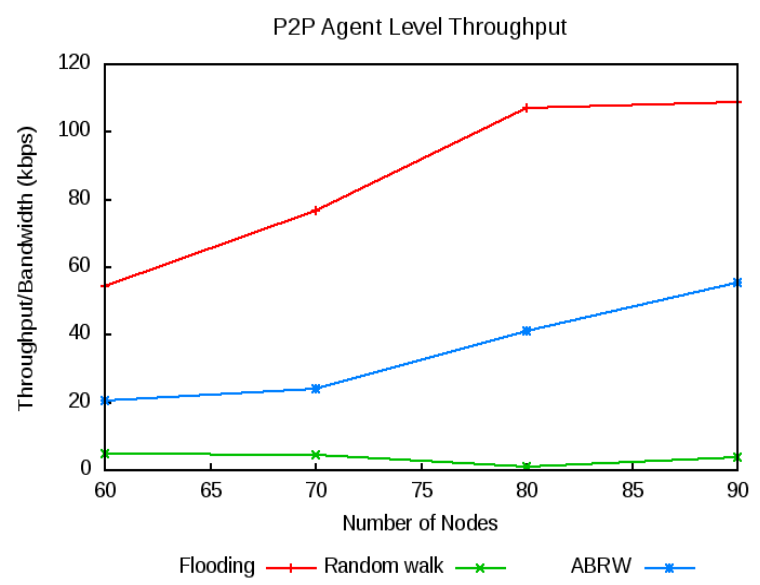

Figure 8. Network Bandwidth

A good resource discovery protocol consumes less bandwidth (i.e. less throughput) and also should provide equally good success rate. Figure 8 shows comparisons of throughput for various protocols. Even though the existing method consumes lowered bandwidth, but then there is a tradeoff in terms of success rate. Our version of random walk protocol is the best among the other compared ones.

\section{CONCLUSION}

Efficient and quick resource discovery algorithm is the need of the hour. For an unstructured Mobile Peer-to-Peer network the situation becomes more difficult due to mobility and energy constraints. With these limitations adopting traditional P2P resource discovery protocol directly over MANET is undesirable. In this paper a random walk based resource discovery mechanism for such dynamic network has been studied and analyzed. In our work, we discuss few open problems for random walk based resource discovery under MANETs. The observed drawbacks have been improved by our suggested technique. To further improve the random walk mechanism we have to incorporate a quasi-flooding based hybrid approach in it. Our future work will focus on improving the algorithm using an hybrid approach with dynamic TTL adaptation and mixture of long jumping scheme.

\section{REFERENCES}

[1] C. Gkantsidis, et al., "Random walks in peer-to-peer networks," Twenty-third Annual Joint Conference of the IEEE Computer and Communications Societies INFOCOM, vol. 1, pp. 1-12, 2004. 
[2] A. Mian, et al., "Identifying Open Problems in Random Walk based Service Discovery in Mobile Ad hoc Networks," IICS, vol. 165, pp. 91-102, 2010.

[3] A. Helmy, "Efficient resource discovery in wireless AdHoc networks: Contacts do help," Chapter in: Resource Management in Wireless Networking Series: Network Theory and Applications, pp. 419-471, 2004.

[4] R. Schollmeier, "Protocol for peer-to-peer networking in mobile environments," in Proceedings of the 12th international conference on computer communication and networks, pp. 121-127, 2003.

[5] S. Androutsellis-theotokis and D. Spinellis, "A Survey of Peer-to-Peer Content Distribution Technologies," ACM Computing Surverys (CSUR), vol/issue: 36(4), pp. 335-371, 2004.

[6] M. W. H. D. Choi and H. H. Park, "An Enhanced Gnutella for Ad- Hoc Networks," International Conference on Systems and Networks Communications, ICSNC, vol. 3, 2006.

[7] M. Gerla, et al., "P2P MANET's-New Research Issues," in Peer-to-Peer Mobile Ad Hoc Networks, volume 05152 of Dagstuhl Seminar Proceedings, Internationales Begegnungs- und Forschungszentrum für Informatik (IBFI), Schloss Dagstuhl, Germany, pp. 1-25, 2005.

[8] V. Kalogeraki, "A local search mechanism for peer-to-peer networks," Proceedings of the eleventh international conference on Information and knowledge management, pp. 300-307, 2002.

[9] Z. Zhuang, et al., "Hybrid periodical flooding in unstructured peer-to-peer networks," in International Conference on Parallel Processing, 2003.

[10] R. Gaeta and M. Sereno, "Generalized Probabilistic Flooding in Unstructured Peer-to-Peer Networks," IEEE Transactions on Parallel and Distributed Systems, vol/issue: 22(12), pp. 2055-2062, 2011.

[11] Q. Lv, et al., "Search and replication in unstructured peer-to-peer networks," in Proceedings of the 16th international conference on Supercomputing ICS, pp. 84-95, 2002

[12] I. Jawhar and J. Wu, "A Two-Level Random Walk Search Protocol for Peer-to-Peer Networks, pp. 1-5.

[13] Y. Chawathe and S. Ratnasamy, "Making gnutella-like p2p systems scalable," Proceedings of the conference on Applications, technologies, architectures, and protocols for computer communications SIGCOMM, pp. 407-418, 2003.

[14] T. F. O. Ratsimor, et al., "Allia: alliance-based service discovery for ad-hoc environments," in Proceedings of the 2nd international workshop on Mobile commerce WMC, pp. 1-9, 2002.

[15] S. Jiang, et al., "Lightflood: an efficient flooding scheme for file search in unstructured peer-to-peer systems," in Proceedings of International Conference on Parallel Processing, pp. 1-9, 2003.

[16] S. Jiang and L. Guo, "Light Flood: Minimizing Redundant Messages and Maximizing the Scope of Peer-to-Peer Search," IEEE Transactions on Parallel and Distributed Systems, vol/issue: 19(5), pp. 1-14, 2008.

[17] T. Lin, et al., "Dynamic Search Algorithm in Unstructured Peer-to-Peer Networks," IEEE Transactions on Parallel and Distributed Systems, vol/issue: 20(5), pp. 654-666, 2009.

[18] S. M. Thampi, "Elephants Journey towards Successful Resource Discovery in Unstructured P2P Networks."

[19] M. Shojafar, et al., "An efficient and distributed file search in unstructured peer-to-peer networks," Peer-to-Peer Networking Application, 2013

[20] C. Wei, "Intelligent Search Technology Combining Semantic Grid and Clustering," TELKOMNIKA Indonesian Journal of Electrical Engineering, vol/issue: 11(8), pp. 4803-4809, 2013.

[21] J. Han and S. Zhang, "Using the Reputation Score Management for Constructing Fair P2P File Sharing System," TELKOMNIKA Indonesian Journal of Electrical Engineering, vol/issue: 11(6), pp. 3200-3205, 2013.

[22] B. Shah and K. I. Kim, "Towards Enhanced Searching Architecture for Unstructured Peer-to-Peer Over Mobile Ad Hoc Networks," Wireless Personal Communication, vol/issue: 77(2), pp. 1167-1189, 2013.

[23] K. S. Bok, et al., "A Resource Discovery with Data Dissemination over Unstructured Mobile P2P Networks," KSII Transactions. Internet Information. Systems, vol/issue: 6(3), pp. 815-835, 2012.

[24] L. Zhang and J. Liu, "Efficient Search Scheme in Mobile Peer-to-Peer Network," in International Conference on Industrial Control and Electronics Engineering, pp. 1235-1238, 2012.

[25] J. Liu, "The Research of Resource Searching Algorithm in Mobile P2P Network," in Fourth International Conference on Computational and Information Sciences, vol. 1, pp. 811-814, 2012.

[26] T. P. Nghiem, et al., "Bichromatic Reverse Nearest Neighbors in Mobile Peer-to-Peer Networks," IEEE International Conference on Pervasive Computing and Communications (PerCom), pp. 159-164, 2013.

[27] M. K. Sbai, et al., "A Membership Management Protocol for Mobile P2P Networks," in Mobility '09 Proceedings of the 6th International Conference on Mobile Technology, Application \& Systems, 2009.

[28] R. Beraldi, "Random walk with long jumps for wireless ad hoc networks," Ad Hoc Networks, vol/issue: 7(2), pp. 294-306, 2009.

[29] D. N. da Hora, et al., "Enhancing peer-to-peer content discovery techniques over mobile ad hoc networks," Computer Communications, vol/issue: 32(13-14), pp. 1445-1459, 2009.

[30] M. Singh, et al., "Resource Discovery Using Mobile Agents," 2010 Fifth Int. Conf. Front. Comput. Sci. Technology, pp. 72-77, 2010.

[31] H. Wu, et al., "A resource discovery strategy for mobile peer-to-peer networks," in Proc. of International Conference on Wireless Information Networks and Systems, pp. 42-46, 2010.

[32] D. Qu, et al., "Research on locality for resource discovery over mobile P2P networks," IET Int. Commun. Conf. Wirel. Mob. Comput. (CCWMC), pp. 425-428, 2009.

[33] J. H. Lee, et al., "An Energy-Effective Routing Protocol for Mobile P2P Systems," 4th Int. Symp. Wirel. Pervasive Comput.ing, pp. 1-5, 2009.

[34] P. D. Linh, and T. Sato, "Performance Evaluation for GIA based P2P File Sharing in Mobile Cellular Networks," 2010. 
[35] J. Han and K. Lee, "Mobile pee-to-peer systems using super peers for mobile environments," in Proceedings of International Conference on Information Networking, pp. 1-4, 2008.

[36] D. Han and J. Zhang, "An Optimized Gnutella-like P2P Protocol in Mobile Networks," Journal of Networks, vol/issue: 7(9), pp. 1464-1471, 2012.

[37] A. Arunachalam and O. Sornil, "Issues of Implementing Random Walk and Gossip Based Resource Discovery Protocols in P2P MANETs \& Suggestions for Improvement," Procedia Computer Science, vol. 57, pp. 509-518, 2015.

[38] K. Oikonomou, "A Study of Information Dissemination Under Multiple Random Walkers and Replication Mechanisms," pp. 118-125, 2010.

[39] C. E. Perkins, et al., "Ad-hoc On-Demand Distance Vector Routing," Proceedings of Second IEEE Workshop on Mobile Computing Systems and Applications, WMCSA, pp. 90-100, 1999.

[40] A. Arunachalam and O. Sornil, "An Analysis of the Overhead and Energy Consumption in Flooding, Random Walk and Gossip Based Resource Discovery Protocols in MP2P Networks," Fifth International Conference on Advanced Computing \& Communication Technologies (ACCT), pp. 292-297, 2015. 